\title{
Architectural historical heritage: a tridimensional multilayered cataloguing method
}

\author{
DOTT. ARCH. DANIELE CALISI ${ }^{*}$, DOTT. ARCH. ALESSIO TOMMASETTI ${ }^{* *}$, ARCH. ROSA TOPPUTO *** \\ * UNIVERSITÀ DEGLI STUDI DI ROMA TRE \\ Via Rimini 14, 00182 Roma, Cell. 335 6586554, d.calisi@gmail.com - www.darc-studio.eu \\ ** UNIVERSITÀ DEGLI STUDI "SAPIENZA" DI ROMA \\ Via Don Filippo Rinaldi 16, 00181 Roma, alessio.tommasetti@gmail.com - www.darc-studio.eu \\ *** Via Palermo 8, 70024 Gravina, rosa.topputo@ gmail.com - www.darc-studio.eu
}

KEY WORDS: Database, 3D Modeling, Digital Architecural Multilayered Archive, Augmented Reality, Image Based Modeling

\section{ABSTRACT:}

In the Future the digital filing system will be the method for storing and cataloguing heritages, private assets and arts collections.

Today this elaborate process is confined only to the library, painting or parietal heritage.

What is missing is a digitalized acquisition of the architectural heritage, which is described at multiple levels of representation.

Taking a critical look at the urban setting until you reach the single buildings in their complexity, there is a clear need to establish an open and up-to-date system in order to communicate the different degrees of interaction with the architectural elements that must be preserved and accessed to like a work of art.

The breakdown and cataloguing at tridimensional levels affects the different scales of the representation of the city at the stage of stimulating and interactive fruition for those users interested in historical and cognitive research and at the stage of active and project implementation well.

The hierarchy of layers of data storage city based should be lived and experienced on a superficial stage as a simple user of the knowledge offered by the digital language of animation and interactivity. It may be the case of a tourist or a citizen who is eager to deepen his awareness of a building, a neighbourhood together with its layering of history and architectural value.

This article proposes the development of a database that will be used and extended from time to time with new information related to surveys, projects and restorations of the existing.

\section{THE DIGITAL ARCHIVE FOR THE STUDY AND CLASSIFICATION OF HISTORICAL (by Rosa Topputo)}

It is now possible to live and move in the fabric of historic consolidated and stratified cities and study their most interesting features using the new technologies that provide content and containers suitable for the purpose.

The Data Archive is the focus of this trial because it allows to combine in a system of connections all the information needed to give the right meaning to the representation of complex city without having to make compromises of abstraction, due to the lack of support systems to the means of expression.

Already in the planning stage of the concept behind the creation of a digital archive updated and multifaceted has needed to display the management of three-dimensional models and of all information to which they bind, with a graphical user interface easy to understand, yet comprehensive and interactive.

As the archive itself, the interface tools can grow and change, placing them in a hexagonal board that makes geometrically obvious the desire to expand the system.

A digital system in motion, therefore, not a simple twodimensional and three-dimensional data collector.

The continuous development of this idea experimental irrevocably determines the usefulness and longevity.

DAMA, Digital multilayered Architectural Archive, then arises from the need to visually organize the digital archive of $3 \mathrm{D}$ models with a set of interactive nodes that can reduce or increase the weight of information displayed in real time on a single building or neighborhood.

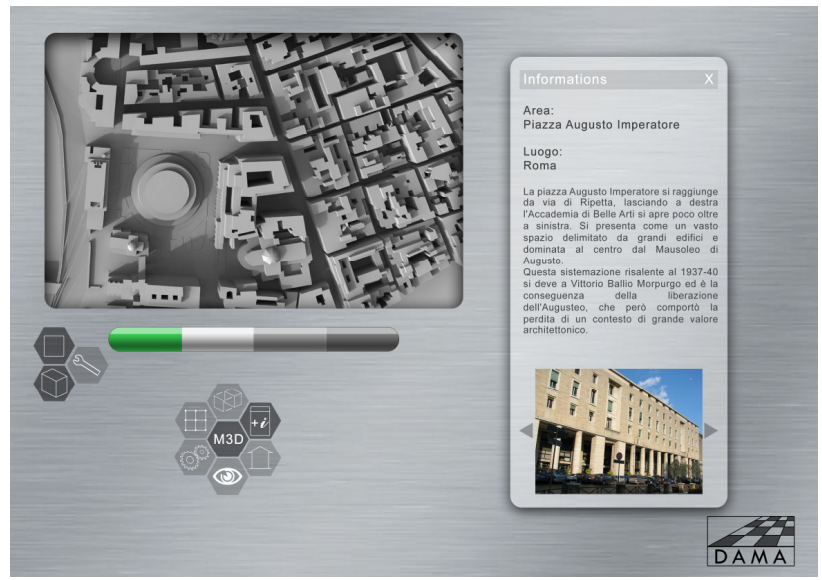

1. DAMA interface at first level, hypertext info

The idea is to break down the massive number of data used to reconstruct as closely as possible the historic architecture in different sub-folders organized by themes: a) the basic threedimensional model that serves as the root node and not be separated; b) applied texture derived from specific photographic 
surveys and diversified by passages of graphic resolution from lowest to highest updated automatically with the approach to the model; c) hypertext information related to the area or building in analysis, consisting of historical and updated photographic footage, historical information, digital movies from real or virtual, legible overlapped in the model that is visible or in the fully functional interface or in its configuration light (light DAMA), installed on devices and portable devices with digital camera and operating system that manages the Augmented Reality; d) technical content such as plans, elevations and sections for understanding of the architectural; e) photo-textured properly screened, representing in some cases integration of specific parts of the model; $f$ ) three-dimensional details to make possible to increase the resolution of the basic model at point a, maintaining the solid nature of the model; g) information derived from other types of models that serve to further integration of the basic model and of the details applied to it.

The nodes will be turned on or off adapting to the different levels of use of the archive, allowing an infinite number of configurations that are able to guide the navigator to the userdesired result. verified by historical perspective and technical compatibility with the system itself, before being entered and made accessible to the community.

update in a properly and effectively way, and, as has been mentioned previously and as will be subsequently developed in later chapters, is the user who decides the degree of interaction with the system.

For subsequent phases, the user will move from awareness of model to the transformability of the same, taking on the relative system of implications and theoretical - scientific design rules. Similarly to what happens to the Data Archive and its interface DAMA, the user also expands their knowledge and awareness in the field of architecture, also motivated by the spirit of competition with rival digital computer, which soon will be transformed into creative collaboration and co-creation. The first level of three-dimensional navigation of the archive will be less detailed from the technical point of view but of a great emotionally impact.

You $\log$ on to a digital model by clicking on the interactive floor plan divided into sections to separately manage a very large amount of three-dimensional data, and choosing the area of complex architecture, historical or contemporary in which the

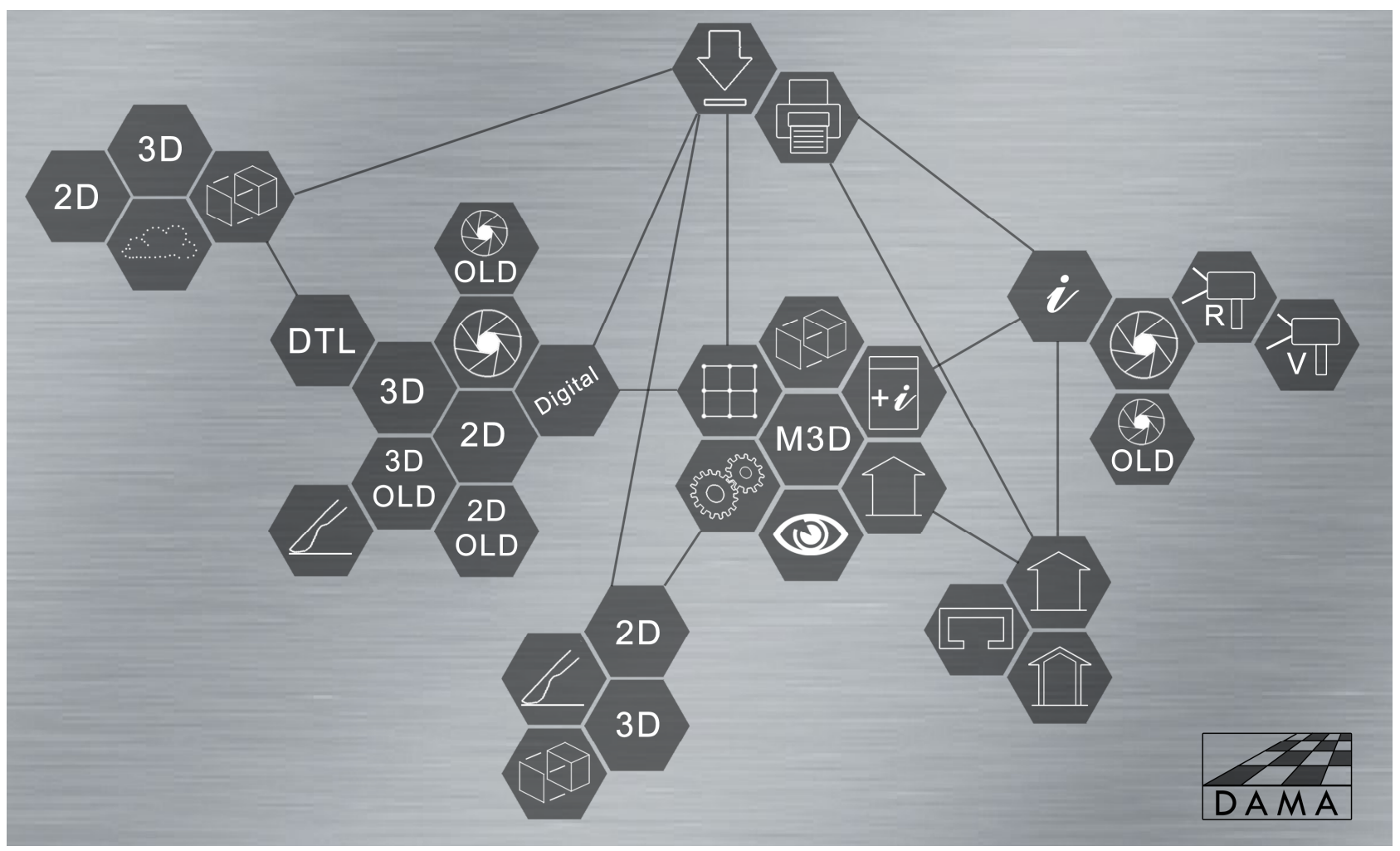

2. DAMA interactive interface with icon nodes

The tools of self-generated toolbar are biunivocally connected to nodes, allowing the dual ability to change the amount of information displayed on the three-dimensional based model directly on the display window, the main layout of the interface, or in the technical area for the modification of settings represented graphically in the nodes diagram.

It is assumed, therefore, a type of open and easily manageable interface with a graphical user-friendly navigation and the addition of nodes for each element that goes to enrich the potential and accuracy of the information provided.

The type of open system and easily updatable is not synonymous with weakness of the instrument or inadequacy of basic sources that feed it: the latter, in fact, will be re-checked, user desires to be immersed.

An experience traveled independently or led by others or supervisors networked with DAMA.

It's the green level as indicated on a bar below the interface, to sign, with a universal language, the accessibility and the immediate recognition of commands to the virtual experience within the area examined.

The use in this phase of the database has an educational and training aim, exploitable in the faculties of Architecture, in museum exhibits or exhibition that require a virtual reconstruction of the architecture, possibly with a tailored user experience immersive. 
The product of this phase is a Real Time navigable model with simplified volumes but with photo-realistic textures, to maintain a close connection with the reality of reference.

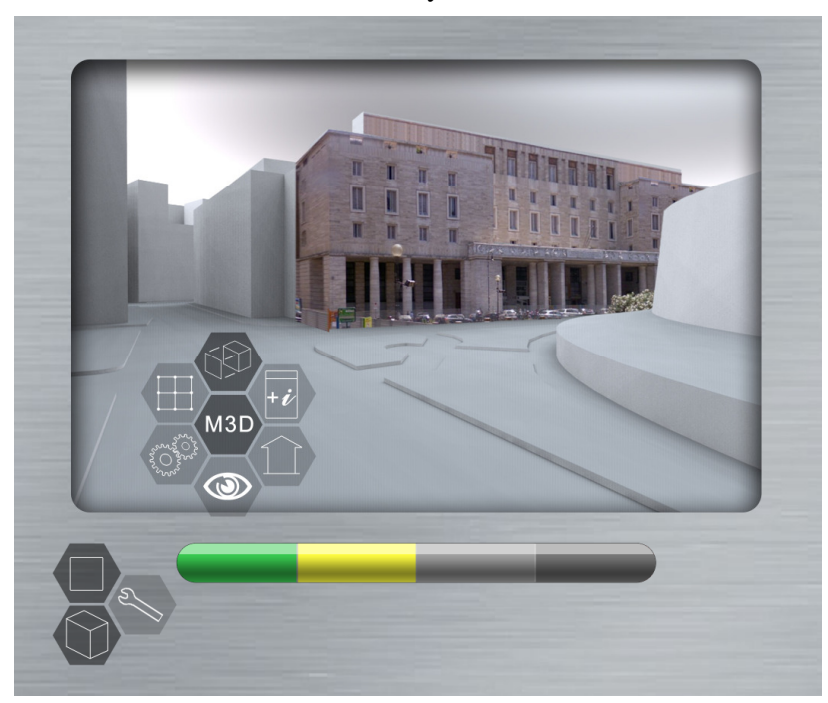

3. Real Time Viewing with photorealistic textures on

Also in real time it's possible to turn off the textures to realize the relationships between spaces and volumes with a neutral base color, or turn on additional information overlay that can satisfy the thirst for knowledge of who will go beyond the simple facade of each building.

At a higher level to the first, a yellow, the student of architecture or the user is particularly interested find the information necessary to learn building techniques and compositions related to architectural construction: detailed three-dimensional digital models can be browsed, compared with the current urban plan, declined in the traditional geometric representations such as plans, elevations and sections.

The transition between the levels is automatic thanks to the ability to recognize the use of toolbars that will adapt to the system of the delivered and displayed content. A structure and statistics code in the heart of DAMA allows flexibility and the change of the interface directly linked to the methodology for using and exploiting the resources of the Data Archive.

The system thus configured allows the appropriate use of resources, without adding hardware and software if not absolutely necessary, and above all without intimidating the user with a vast amount of possibilities so big to not allow the choice of at least one of them.

The second level gives way for scientific discussion between the various users that in the virtual or real field can put theirknowledge on different levels, in an ideal experiential timeline, and understand from the overlaps how to acquire more useful information, but also understand the research paths that determinated them.

The opportunity to grow culturally and scientifically sprung from the active and stimulating confrontation, further animated by the action of creating and editing the tool used for research with update contents, properly filtered and verified.

\section{THE DIGITAL ARCHIVE FOR THE DESIGN OF CONTEMPORARY (by Alessio Tommasetti)}

The analysis and study of complex city inevitably converge in the case of investigating the possibility of a integrated design considering the layers of history and social and cultural background as values existing in the intervention design, and not added or options, reasonably included between the compositional principles.

Contemporary Architecture is an integral part of the historic city, born and developed context and drawing attention to the design spirit and unity of effort that many designers have made previously to create the viable border that has become habit, custom and tradition, or it is proposing completely alternative perspectives of using of spaces, in line this time with the changed needs of the XXI century society.

Whether it's action complied with the Tradition or is strongly projected towards the Future, the design of today need innovative and powerful tools capable of keeping under control every aspect of planning view and update to a more appropriate level of representation for information and content.

The observation, understanding and awareness of what exists, while playing in virtual environment, are the essential basis for planning at the start of the stimuli for the creation of a work is not separated from the space-time continuum fabric and consolidated the social status that houses in it.

The Data Archive proposed in this experience guides the designer not only at the stage of awareness and acquisition of data necessary for the pre-design stage, reducing the time of search and retrieval with the plus of getting marked scripts and verified by the competent bodies linked to the archive and ready in the most common digital formats, but also during the conception, development and communication of the digital project.

In the third level, then, the moment academic and theoretical, as described in the previous chapter, leave space at the time of implementation, right in the field, giving users the volumetric and metrics ratios to design and place an ex novo project and determine the conflict of that work with surrounding urban fabric.

It 's the Orange level dedicated to more experienced user with different needs than students of Architecture, and contains all the retrievable and customizable processing to set two dimensional and three-dimensional drawings of areas already in the archive.

The system nodes considered at this moment are linked together to form complex chains with three-dimensional models of urban-scale volumetric detail, that can be increased up to architectural one to display in row the model parts useful in the compositional creation.

Real-time DAMA shows icons to change the level of detail of the models in the project area, allowing navigation of the virtual space as a whole or to isolate the parts on which to focus the most attention.

The "smart" icons system makes easy to use of certain operating tools and determines the choices' extension by agreeing to the working methodology of the individual user, starting with a basic layout of hexagonal cells in automatic expansion.

The proposed visualization allows you to move easily between different levels of synthesis of the existing reality performed on the three-dimensional model to make it suitable for also stylistically very different project initiatives.

It is possible, indeed, maintain as active a uniform gray color of the basic material applied to the three-dimensional scenes in the navigation window of the system allowing you to focus more easily empty and full of architectural space, thus emphasizing the very complex volumetric articulation in historic cities.

The monochromatic full, though made by a visually compelling global illumination lighting algorithm, as opposed to a background in that spot is empty, lets focus on some design choices in a way universally recognizable and understandable. 
Activating the button of the advanced texture, the threedimensional model adheres to reality in a concrete way showing the high-resolution photo carefully applied to volumes, obtained through a procedure that combines photo-rectification to View Depending Mapping and Render to Texture technique on each single item for which you hold the appropriate degree of information obtained with the aid of photographic campaigns planned specifically for the enrichment of the Data Archive. This display mode is useful for the choice of colors and materials of the ex novo project with respect to pre-existence, such as a color palette that will bring the project to a choice of complementary or contrasting with the urban context having all the detailed information contained in intervention area.

Acting on the details icon button you increase the complexity of three-dimensional model showing details such as doors, moldings, decorative or structural elements, obtained from a three-dimensional modeling hybrid integrated with the photographic technique to compensate for any shares in the process of completion and review.

A model articulated in this way is light in weight, in bytes, and can be managed very quickly during visualization and creating render images, and thus acquire information and complexity in different types of user-defined operations.

As if each individual application's demand on the virtual model altered to bring about a different result, and, potentially, the exact same model could generate infinite copies of itself declined in developed for other purposes classified by sector.

A number of dedicated controls allow in the third level of DAMA to obtain all the deliverables required for the project to obtain two-dimensional geometric and metrics information on the buildings belonging to the archive, not like a fixed projection but freely editable: by simply moving in space horizontal or vertical slice planes, sections are obtained as vector drawings at the project points of interest, while moving the slice plans outside the three-dimensional block elevations can be saved in vector or high resolution raster files in, containing materials and textures with color swatches. Moving into perspective virtual area, the Real Time rendering engine processes only that which is framed by the user by recognizing the movement of elements which interact with the space to highlight and display more accurately, speeding and facilitating the user's immersive reality with the possibility to save highresolution print screens such as static images or movies at any time.

And according to the display options listed above, the animated tale takes on entirely different stylistic features and is linked to the expressive willingness of the director but also to the receptive one of the interlocutor, which should interact with the work and presenting it in bureaucratic decision making, and often does not understand so-called classical technique drawings as layouts, elevations and sections.

A contribution of considerable magnitude for the final stage of architectural design that is communication, whereas the experienced user can upload their three-dimensional model within the archive, and interacting with the interface, set the project proposal in a realistic and detailed environment thanks to the already defined three-dimensional fabric in the intervention area.

In the early stages of architectural design, a direct access on a field of interest using the simplified version of the interface, DAMA Light, allows the user to simply frame buildings pointing at them with a smart device provided with a digital camera and an operative syst

em supporting Augmented Reality, and store notes, information and possibly outline the project first idea working on-screen overlay the real environment.

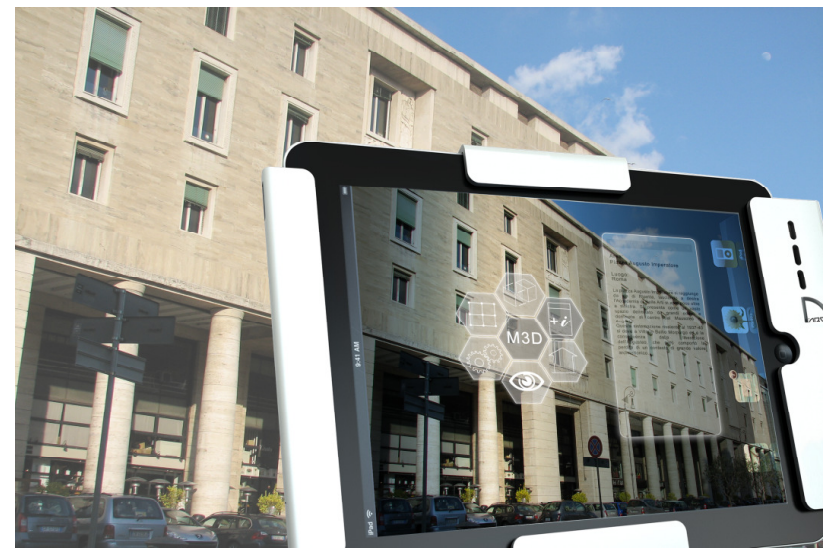

4. DAMA light for smart devices

In the case of an architectural competition of considerable resonance at social and cultural level, the various proposals may have a common and consistent basis insertion and become an integral part of the Data Archive in a section especially created and visible from all interested users.

The three-dimensional models can be downloaded and used outside the Data Archive interface, and the more interesting possibility is their updating made by the user themselves, uploaded after inspection and verification of consistency by historical and architectural requirements, and placed in the DAMA environment.

In this way we can ensure a continuous updating of threedimensional data is for new projects of contemporary architecture that will change the structure of the urban fabric, both for maintenance work on existing models already under study and belonging to the digital archive.

It reaffirms the importance of the Data Archive as essential tool to the architectural story, as a social phenomenon linked to anthropogenic space that changes continuously and precisely for this reason it needs an innovative system, flexible and upgradeable over time not only by computer database technicians but also by external users who use it as a everyday work tool.

The development and integration of information sources of reference and the exponential improvement in the acquisition and production techniques of these final results qualify the digital archive enriching the areas of application projects, which range from Cinema and Animation set design to arrive at the Augmented Reality and the Holographic Projection viewing, of course, passing through the increase in the degree of adherence to the reality of the traditional computing model.

\section{THE DIGITAL ARCHIVE FOR THE RESTORATION (by Daniele Calisi)}

The last level for a coherent and comprehensive digital archive of architecture necessarily has a wealth of information and data that increase the permeation of information, lack of representation, the number of visual or perceptive variables, but also the complexity of the interface to the 'user that could choose what and how check the information on the built environment.

First, following the process at all stages, it is clear that, at this stage, the user can access to information on individual buildings. The database available to the user contains, on one hand, the information that can also be found in the previous levels, and on the other hand more detailed information depending on the type of user. 
An ordinary tourists will have access to historical notes, curiosities, critical boards on the quality of architecture, possible reconstructions of ancient monuments and ruins, or even information and details of pieces of art that one particular building might contain.

Other users may, instead, be more interested in architectural forms, which in this fourth level are shown in a model certainly more detailed than those in previous stages. From the Image Based Modeling must necessarily pass through a modeling that comes by an analysis of the architecture on which has made a survey with the right tools: direct survey; by straightening photographs; or by the latest methods, as for example the laser scanner.

Such a digital model can be exploited by many users but it is definitely a more useful instrument for technicians and designers who can utilize it in many and different ways relevant to architecture's aims: historical analysis; possibility of obtaining elevations and sections; formal perception of the building and its weight in the continuity of the street fronts, and so on.

Several times we have insisted on the chance of having a database updatable on different levels, and this possibility becomes even more effective at this stage, in which the added information are provided mainly by technicians, architects and engineers who have carried out investigations, surveys or projects on the building itself.

It could also be interesting to imagine a close collaboration with the universities where, for decades, surveys and analysis on the existing architecture were produced in specialized classes: in this way, the archive could also be a database from which to draw and verify which themes are still to be developed, or which ones are to be updated, or also implemented, and, at the end, insert the final results of the research to DAMA.

However, the information provided in the fourth level must necessarily be at different representation complexity. The three-dimensional digital model, for weight's reasons, should be simplified in order to be navigable by any user, and, unfortunately, the simplification is greater the more structured is the building, whether of the feature shapes that the ornaments.

Clearly, the harder simplification must be done first for the historic building, where dripstone and moldings, digitally created in three dimensions, risk to make unmanageable the navigability and the use of the 3D model.

Not all users have at their disposal devices suitable to orbit, make sections, or steer a complex model, while the idea of a digital archive was created precisely to allow everyone to access at more and more detailed information on the existing buildings. The manageability of the information, on which ones provide and on the way to represent them, is perhaps the main problem to be defined.

The matter could be solved using the practice of intervention on the buildings and also the fact that, at the end, a digital archive (and a digital representation) is not necessarily only related to a three-dimensional modeling.

Actions of consolidation or restoration on the existing buildings, in practice, do not require the three-dimensional modeling, which, although offers a comprehensive view of the object, cannot provide dimensional information, that can only be drawn from sections or elevations in true form, ie twodimensional drawings.

Moreover, the digital representation encloses many areas of design, modeling and photography just to name those most related to the built, but also extends to the field of graphics and communication if we consider the ways to propose something as a product (advertising, marketing, design or architecture).
Definitively, on the one hand we have the consciousness that an increasing detail at low scales of representation is more useful to a restorer than to a designer who use the representation of detail only for the executive drawings of new buildings (he needs instead the environment at major scales to study the context and relate his project to that).

On the other hand, the detail of architectural ornaments does not need to be represented also in the three-dimensional model, where it may be simplified or represented by the Image-based modeling.

It could be part of a parallel database, that complete and interpenetrate with the 3D model, and can include: twodimensional digital drawing; point clouds resulting from any survey campaigns with laser scanners that provide a detailed documentation in particular of those ornaments that have been decayed by external factors, such as capitals, statues, molding and so on; high resolution photographic documentation that attests to the closer aspect of the building to reality in a given historical period.

Other information concern on additional studies on the building could also add to these documents specifically related to the formal aspect of architecture, as for example: an historical analysis that can also be represented graphically on a threedimensional mapping model; or deterioration patterns given in $2 \mathrm{D}$ boards; the accessibility to the original plans that provide an effective and essential tool to analyze the work and the changes over the centuries; a set of photos or even historical sights are also useful in understanding the evolution of the context and relationships between buildings.

The 3D model, its sections, or its projection, may contain landmarks that could indicate the presence of further details and information, which can also be updated and posted by the users.

However another question has to be define too: at this stage, who can have the opportunity to upload his own new research to DAMA?

The peculiarity of the information to upload, detailed and specialized, and the guarantee of high quality of the information, require a limitation at this stage to only a few users allowed to upgrade the database.

An anonymous user could not guarantee a high level or even the exact correspondence of his studies to reality. It is necessary that the subjects delegated to uploading data are authorized by the system (DAMA), or provided of a professional contract by administrations and, finally, by academics who have made specialized investigation on a particular building in their classes.

All information entered will be retrieved through an interactive interface, and made available to anyone who had need to query the archive.

The potentiality of a digital database, however, is not limited to providing information to an individual user. Note that one of the main features of this system is to be able to update the knowledge on a building, but did not delete those immediately preceding.

The data provided on a particular property over the years, offer an interventions and analysis history carried out, allowing to better understand the existing under three main aspects.

First, the formal and architecture development, the most obvious, if we imagine to have the archive of 3D models which capture the building in different historical periods.

Secondly, to highlight the deterioration and decay over the decades, through the use of accurately mapping performed on the wall surfaces, infrared patterns to underline the status of structures, or point clouds from laser scanners, where detailed and properly processed and mapped with the photographs, 


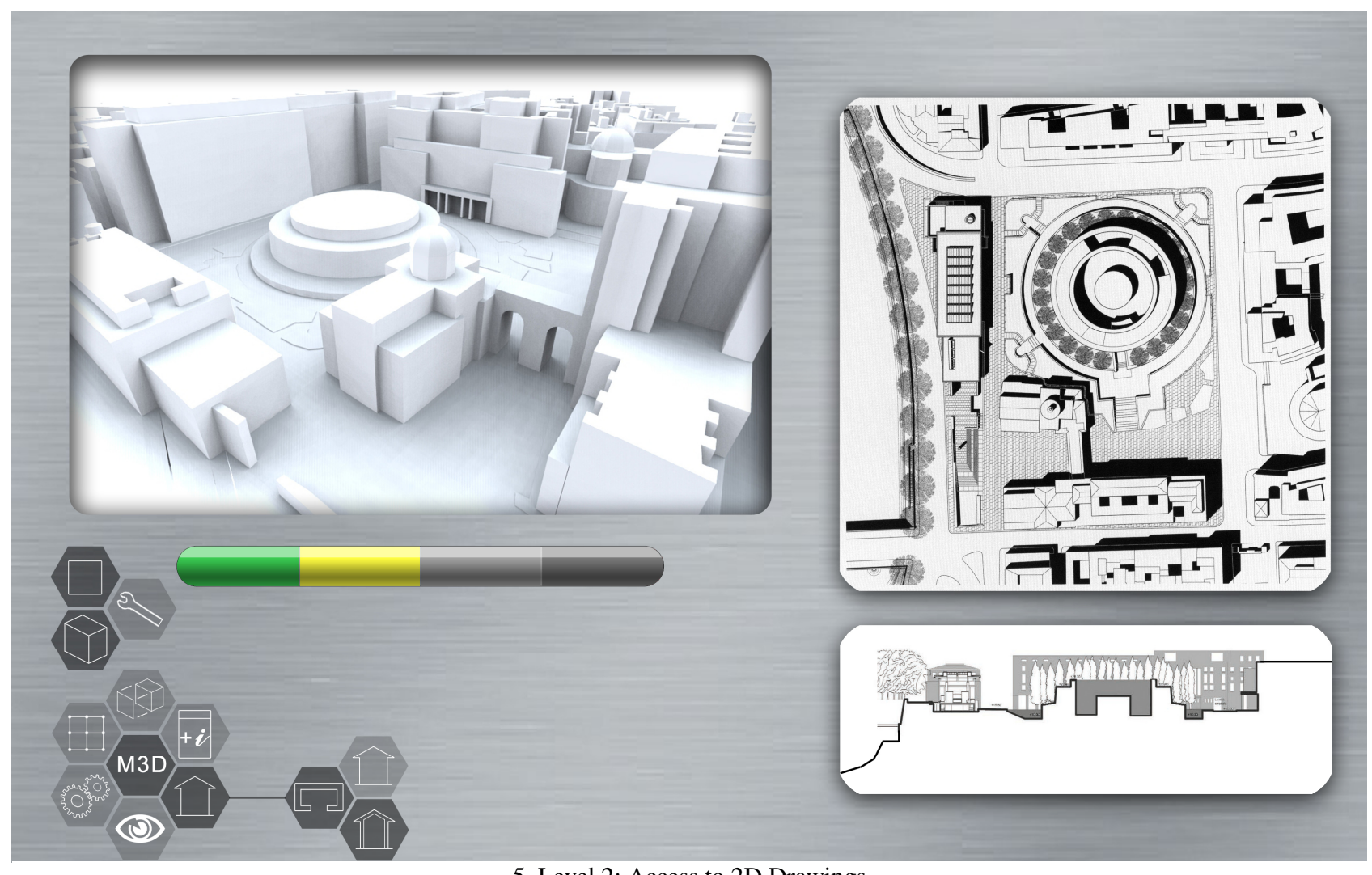

5. Level 2: Access to 2D Drawings

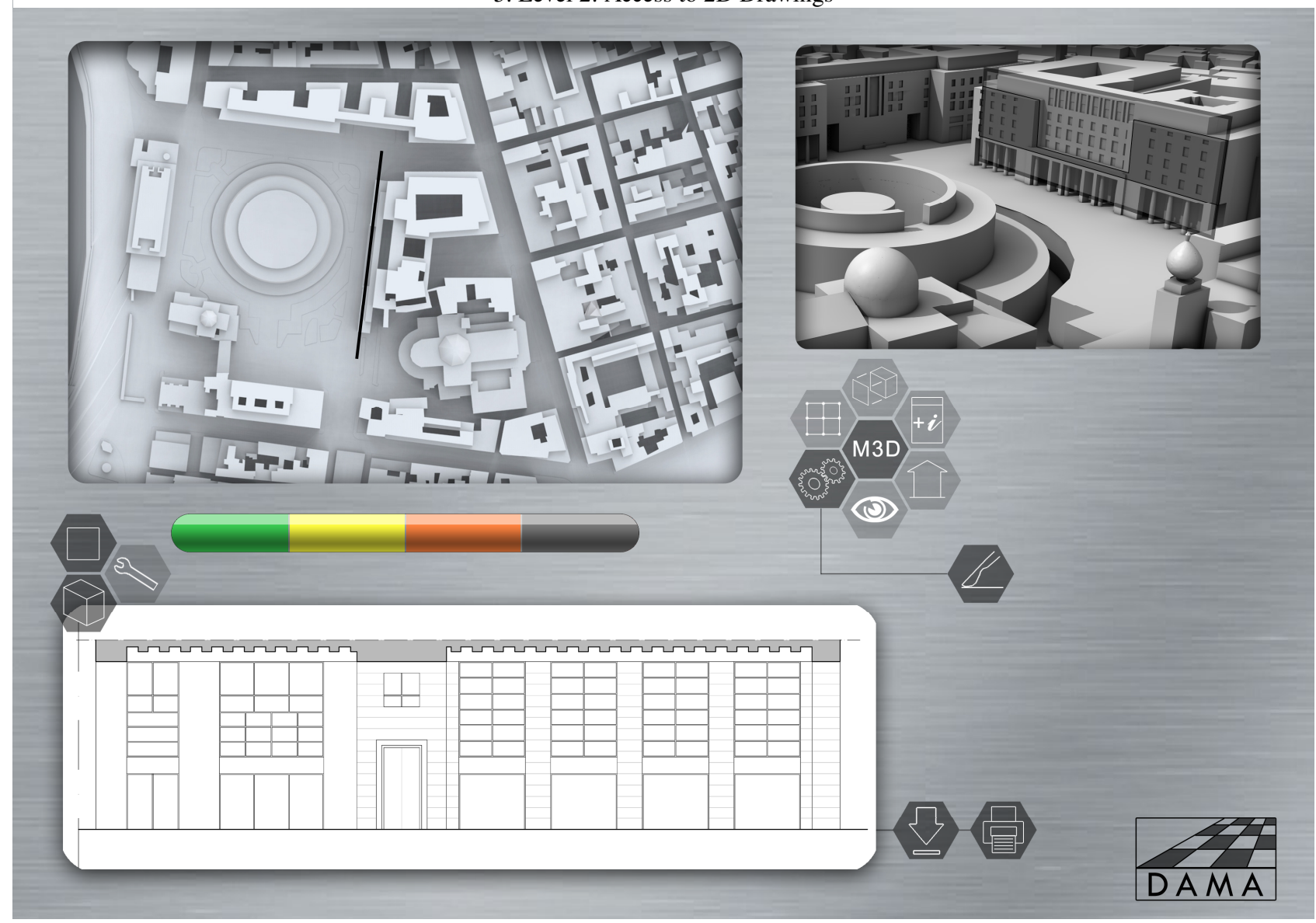

6. Level 3: Generating custom user section from 3D model 


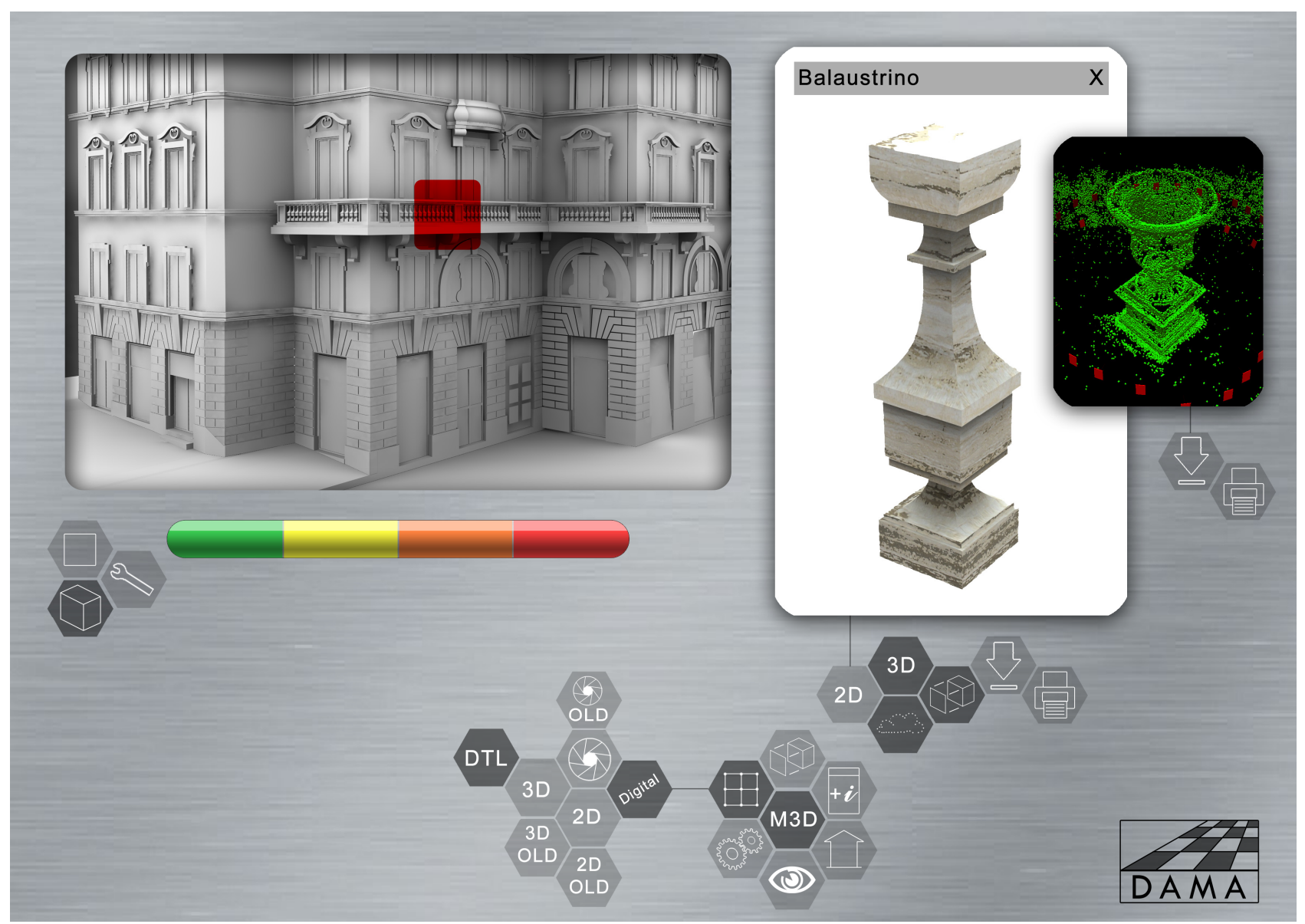

\section{Level 4: Details Viewer choosing on a single building}

would certainly have a very reliable image of the decorative elements.

And last aspect is also able to show the changes in the method of representation, both for those buildings where you can go back to the original drawings directly comparable with the digital survay, both for new buildings, where the representation capacity are still contemporary, but could be different or even obsolete when compared to the analysis performed in the near future and with ever more advanced techniques.

Finally there is a further benefit of such an archival system: the possibility to ensure the information not only to the existing buildings, but also to the property demolished.

Especially abroad, it was common demolish entire buildings, or parts of them (even in city centers), to make way for new buildings that also change the urban environment and the perception of it.

The database would provide the necessary information about the buildings demolished, depending on the size and weight that they had in history, the formal perception limited to the property through the three-dimensional views, but (returning to previous levels of the database) the perception of the urban space as it was before the dismantling.

In Italy, where in fact it is not conceivable, for various historical reasons, a normal demolition activities except for cases of illegal or irreversible decay of the buildings, DAMA would still be useful to provide historical analysis and visualization of entire neighborhoods demolished in the story, or portions of the city torn for reasons of practicability or simply following the bombing in World War II.
There would be many cases, only in Rome for example we could quote the entire Alessandrino neighborhood demolished to give place to Via dell'Impero during the fascist age, or the demolition of the old town to make way for Corso Vittorio Emanuele, or finally to the famous bombing of the San Lorenzo district, which have greatly changed the look.

\section{CONCLUSION AND FUTURE WORKS}

DAMA is an experimental database, it's an idea born from three young architect that work on the contemporary and on the historical fabric, researching the best way to help technicians to improve and speed their job.

An idea that has to be enhanced thanks to upper institutional bodies and the interaction between them: University, the Ministries, City Hall and Soprintendeza.

Will be a long and arduous road before reaching a conclusion, but it will be a great goal for the world community having such a system for all the benefits that DAMA can give in the future.

\section{BIBLIOGRAPHY}

- Daniele Marini - Maresa Bertolo - Alessandro Rizzi, Comunicazione visiva digitale fondamenti di eidomatica, Addison-Wesley, Milano 2001. 
- Marco Gaiani, Metodi di prototipazione digitale $e$ visualizzazione per il disegno industriale, l'architettura degli interni e i beni culturali, edizioni Poli.Design, Milano 2003.

- James Steele, Architettura e Computer - azione e reazione nella rivoluzione del progetto digitale, Gangemi editore, Roma 2004.

- Donald A. Norman, Emotional design - perchè amiamo (o odiamo) gli oggetti della vita quotidiana, Apogeo, Milano 2004.

- H.P. Moreton and C.H. Sequin, Functional Optimization for Fair Surface Design, Computer Graphics, vol. 26, no. 2, 1992, pp. 167-176.

- R. Schneider and L. Kobbelt, Geometric Fairing of Irregular Meshes for Free-Form Surface Design, Computer Aided Geometric Design, vol. 18, no. 4, 2001, pp. 359-379.

- O. Karpenko, J.F. Hughes, and R. Raskar, Free-Form Sketching with Variational Implicit Surfaces, Eurographics, Computer Graphics Forum, vol. 21, no. 3, 2002, pp. 585-594.

- T. Igarashi, J.F. Hughes, Smooth meshes for sketch-based freeform modeling, in ACM Symposium on Interactive $3 D$ Graphics, 2003, pp. 139-142.

- P.A.C. Varley et al., A Two-Stage Approach for Interpreting Line Drawings of Curved Objects, Proceedings Eurographics Workshop Sketch-Based Interfaces and Modeling, Eurographics, 2004.

- O. Karpenko, J.F. Hughes, and R. Raskar, Epipolar Methods for Multi-View Sketching, Proceedings Eurographics Workshop Sketch-Based Interfaces, Eurographics, 2004.

- T. Igarashi, T. Moschovich, J.F. Hughes, As-rigid-as-possible shape manipulation. ACM Trans. Graph. 24, 3, 2005, pp. 1134-1141.

- M. Masry, D.J. Kang, and H. Lipson, A Freehand Sketching Interface for Progressive Construction of $3 D$ Objects, Computers and Graphics, vol. 29, no. 4, 2005, pp. 563-575.

- K. Das, P. Diaz-Gutierrez, and M. Gopi, Sketching FreeForm Surfaces Using Network of Curves, Proceedings Eurographics Workshop Sketch-Based Interfaces and Modeling, Eurographics, 2005.

- L.B. Kara, C. D'Eramo, and K. Shimada, Pen-Based Styling Design of 3D Geometry Using Concept Sketches and Template Models, Proceedings ACM Solid and Physical Modeling Conference, ACM Press, 2006, pp. 149-160.

- L.B. Kara, K. Shimada, Sketch-based 3D shape creation for industrial

styling design, IEEE Computer Graphics and Applications 27, 1, 2007, pp. 60-71.

- Yendo, T., KAWAKAMI, N., AND TACHI, S. 2005. Seelinder: the cylindrical lightfield display.

In SIGGRAPH '05: ACM SIGGRAPH 2005 Emerging technologies, ACM Press, New York, NY, USA, 16.
- Zwicker, M., MATUSIK, W., DURAND, F., AND PFISTER, H. 2006.

Antialiasing for automultiscopic $3 D$ displays. In Rendering Techniques

2006: 17th Eurographics Workshop on Rendering, 73-82. 